\title{
O GENERO CHEILANTHES SWARTZ EM PORTUGAL
}

\author{
M. L. ROCHA AFONSO
}

RESUMO: A A. estudou minuciosamente os espécimes de Cheilanthes spp. existentes nos principais herbários portugueses (COI, LISI, LISU e PO) e concluiu que grande parte dos espécimes em tempos identificados como Ch. pteridioides ou Ch. fragrans não pertenecem de facto a esta espécie mas sim ás afins Ch. guanchica C. Bolle, Ch. maderensis Lowe e Ch. tinaei Tod. São apresentadas chaves de identificação para as espécies portuguesas, bem como listas dos exemplares estudados e respectivos mapas de distribuição.

RESUMEN: Hemos estudiado minuciosamente las especies de Cheilanthes ssp. existentes en los principales herbarios portugueses (COI, LISI, LISU e PO), concluyendo que la mayor parte de estas, identificadas anteriormente como Ch. pteridioides o Ch. fragrans, no pertenecen de hecho a esta especie sino a aquellas afines, Ch. guanchica $\mathrm{C}$. Bolle, Ch. maderensis Lowe e Ch. tinaei Tod. Se presentan unas claves de identificación para las especies portuguesas, así como listas de los ejemplares estudiados y los correspondientes mapas de distribución.

O género Cheilanthes Swartz tem sido modernamente considerado (A.C. Jermy \& H.P. Fuchs in Tutin \& al., Fl. Eur., 1: 10. 1964; J. Franco \& M.L. Rocha Afonso in Franco, Nova Fl. Port., 1: 11-12. 1971) como estando representado em Portugal por quatro espécies.

Porém, em revisão recente do género para Espanha ( $C$. Saénz de Rivas \& S. Rivas-Martínez, Lagascalia, 8 (2): 215-241. 1979), verifica-se que parte dos espécimes espanhois em tempos identificados como Ch. pteridioides (Reichard) C. Chr. não pertencem a esta espécies mas devem antes ser referidos a $\mathrm{Ch}$. guanchica Bolle, $\mathrm{Ch}$. maderensis Lowe e Ch. duriensis Mendoça \& Vasc.

O conhecimento deste trabalho espanhol levou-nos a pensar que seria útil proceder a uma revisão do material português a fim de verificar se haveria motivo para idênticas alterações. Assim, estudámos pormenorizadamente os espécimes preservados nos herbários do Instituto Superior de Agronomia, Lisboa (LISI), Faculdade de Ciências de Lisboa (LISU), Instituto Botânico "Dr. Júlio Henriques", Coimbra (COI) e Instituto de Botânica Dr. Gonçalo Sampaio da Universidade do Porto (PO), e chegamos à conclusão que, de facto, essas espécies recentemente citadas para Espanha também se encontram em Portugal, desconhecedo-se até aqui a sua existência neste pais por deficiência de identificação do material herborizado.

Antes de darmos a relação dos espécimes estudados, com a sua 
distribuição em mapas próprios por cada espécie e notas sobre a sua ecologia, entendemos conveniente apresentar umas chaves que facilitem a identificação das sete espécies que julgamos representarem o género Cheilanthes Swartz em Portugal continental.

1 Segmentos das folhas glabros na páginas inferior.

2 Folhas de ráquis fundamente sulcada, com os bordos alados, esparsamente glandulosa; pseu-

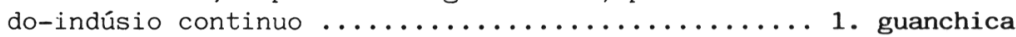

2 Folhas de ráquis com sulco pouco profundo com os bordos não alados, ramentosa; pseụdo-indúsio descontínuo.

3 Pseudo-indúsio fimbriado; segmentos de primei-

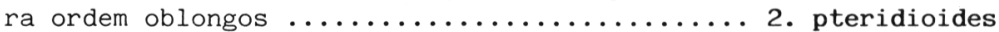

3 Pseudo-indúsio com os lobos inteiros; segmentos de primeira ordem + ovados .............. maderensis

1 Segmentos das folhas ramēntosos na página inferior.

4 Limbo coberto por ramentos filiformes na página superior ........................... vellea

4 Limbo glabro na página superior.

5 Limbo densamente revestido por ramentos clatrados na página inferior ................. marantae

5 Limbo não ramentoso, provido de pêlos glandulosos na página inferior.

6 Limbo \pm esparsamente coberto por pelos curtos $\left(c^{-}\right.$m $2-5$ células $) \ldots \ldots \ldots \ldots \ldots \ldots \ldots \ldots \ldots \ldots \ldots$. tinaei

6 Limbo densamente coberto por pelos compridos (com 5-9 células) .................... 5. hispanica

1. Ch. guanchica Bolle, Bonplandia, 7: 107 (1859).

ALGARVE: Serra de Monchique, Caldas, J. d'Ascenção Guimarães, VI1887 (LISU, P-512); Monchique, A. Moller, VI-1887 (COI).

Esta espécie, só muito recentemente considerada para o Sul de Espanha e Portugal (Saénz de Rivas \& Rivas-Martínez, 1979), parece ser, no nosso País, muito rara e apenas localizada nas faldas meridionais da Serra de Monchique.

2. Ch. pteridioides (Reichard) C. Chr., Ind. Fil., 178 (1905).

BEIRA LITORAL: In rupestribus, ad flum. Mondego, prope Conimbricam, Wilwitsch, VIII-1848 (LISU, P-2616).

ESTREMADURA: Serra de Candeeiros, pr. olhos de Agua, C. Tavares, 29-XII-1953 (LISU, P-55349); Sintra, pr. Penaferrim, J. de Vasconcellos, XI-1951 \& 11-IV-1952 (LISI); pr. Santa Luzia, retro Loires, Welwitsch, II-1853 (LISU, P-2615); Lisboa, Tapada da Ajuda, J. d'Ascenção Guimarães, III-1885 (LISU, P-511); A. Veneno, III-1932 (LISI); Belem (COI); Serra d'Arrábida, C. Romariz 1218, 9-III-1948 (LISU, P-56481); Welwitsch, V-1843 (LISU, P- 2616) \& s/data (LISU, P-2618); in sumis rupibus Serra d'Arrábida, Welwitsch, III-1842 (COI); do Portinho ao Convento, R. Palhinha \& F. Mendes, IV-1908 (LISU, P-2619); Mata do Solitário, A. Rozeira, J. Alte, G. Costa \& A. Serra, 17-IV-1968 (PO 9956); pr. Portinho d'Arrábida (COI); roches à l'est de Portinho, Serra d'Arrábida, J. Daveau, IX-1881 (LISU, P-2612); Serra de S. Luis, alt. 200 m. , J. Daveau, III-1879 (LISU, P-2613). 
RIBATEJO: Serra d'Aire, près de Torres Novas, J. Daveau, VIII-IX1885 (LISU, P-2621 \& 2622); murs de Constancia à Abrantès, J . Daveau 1129, VI-1884 (LISU, P. 2624).

Esta espécie, tal como actualmente definida, tem uma distribuição que corresponde ao Centro Ocidental de Portugal, em zonas calcárias e muros argamassados.

3. Ch. maderensis Lowe, Trans. Cambr. Phil. Soc., 6: 528 (1838).

Ch. fragrans auct. lusit., non (L.) Webb \& Berth. (1847), p.p.

TRAS-OS-MONTES: Bragança, P. Barros Carneiro, 24-III-1944 (P0-6274); Mogadouro, S. Pedro, J.R. dos Santos Junior, I-1925 (PO-2796 p.p.).

ALTO DOURO: Carrazeda d'Anciãi, Ribalonga, J. de Vasconcellos, III1940 (LISI); Régoa, M. Paulino d'Oliveira, V-1879 (COI); muros da Quinta da Vacaria, s/col., 3-III-1879 (LISU, P-2608) \& 1-II1879, s/col., (LISU, P-2609 \& P-2614); Pinhão, entre Ferrão e Gouvinhas, c. $100 \mathrm{~m}$, , P. Silva, Rozeira, Teles \& Rainha, s/data (PO 6709); Urros, Seixo do Vieiro, P. Lopes \& G. Pedro 629, 24IV-1941 (LISI); Figueira de Castelo Rodrigo, Barca d'Alva, estrada para Freixo, A. Rozeira, G. Costa \& J. Araújo, 5-V-1967 (Po 26970); Freixo de Espada-á-Cinta, Quinta do Saltinho, G. Pedro 758-A, 27-IV-1941 (LISI).

DOURO LITORAL: Serra de Valongo, Porto, 2007 (COI).

BEIRA BAIXA: Abas da Serra, Covilhã, A.R. da Cunha, VI-VII-1881 (LISU, P-2611), Castelo Branco, S. Martinho, A.R. da Cunha, VI1881 (LISU, P-2629).

ALGARVE: Serra de Monchique, Picota, 700 m., J. d'Ascenção Guimarães, VI-1887 (LISU, P-512-A).

Esta espécie parece preferir locais abrigados e de clima subcontinental, com solos mais frequentemente xistosos.

4. Ch. tinaei Tod., Giorn. Sci. Nat. Econ. Palermo, 1: 217-218. (1866).

Ch. duriensis Mendonça \& Vasc. Anais Inst. Vinho Porto, 15 (4): 47 (1956).

Ch. fragrans auct. lusit., non (L.) Webb. \& Berth.

Ch. pteridioides auct. lusit., non (Reichard) C. Chr., p.p.

TRAS-OS-MONTES: Miranda do Douro, margens do rio Douro, R. Palhinha \& F. Mendes, VI-1914 (LISU, P-2620); Rozeira \& J. Castro, 13-IV1944 (PO-5288).

ALTO DOURO: Teixeiró, Caldinhas (Baião), P. Lopes \& G. Pedro 58, 22-III-1941 (LISI); Sabrosa, pr. dum ribeiro, A. Rozeira \& J. Castro, 13-II-1945 (PO 4974); Sabrosa, Chanceleiros, A. Rozeira \& J. Castro, 13-II-1945 (PO 4973); Sabrosa, estrada para Gouvães, G. Costa, A. Serra \& Bernardino, 29-IV-1973 (PO 27045); Casais do Douro, Quinta das Carvalhas, Vale das Pombas, G. Barbosa, M. Myre \& G. Pedro 3475, 24-IV-1942 (LISI); S. João da Pesqueira, Soutelo, Monte de Na Senhora de Lourdes, A. rozeira, D. Barreto \& A. Serra, 29-VI-1969 (PO 26971); Ribalonga, J. de Vasconcellos, 
III-1940 (LISI); Carrazeda d'Anciãis, Tua, Tralhariz, G. Barbosa \& M. Myre 3902; 10-V-1942 (LISI); Tua, Fiolhal, G. Barbosa \& M. Myre 3962, 10-V-1942 (LISI); entre a estação de c.f. de S. Mamede de Ribatua e a povoação, G. Barbosa \& M. Myre 3975, 13-V-1942 (LISI); Alijó, S. Mamede do Tua, margem do rio Tua, Rozeira \& J. Castro, 16-IV-1943 (PO 021); Carrazeda d'Aciãis, Foz Tua, A. Rozeira, D. Barreto, G. Costa \& A. Serra, 21-V-1970 (PO 27040) \& 3-V-1971 (Po 27044); entre Régua e Mesão Frio, M. Ferreira, VII-1879 (COI); Régua, Salgueiral, P. Lopes \& G. Pedro 98, 23III-1941 (LISI); Galafura, Ciderma, F. Mendonça \& J. de Vasconcellos 432, 11-IV-1941 (LISI); entre Pinhão e Régua, Rezende, Alexandre, A. Serra \& Bernardino, 8-IV-1976 (PO 27048); Vila Seca de Armamar, Vale do Tedo, A. Mendonça \& J. de Vasconcellos, I1941 (LISI); Valença do Douro; Serro de Sendões, P. Lopes \& G. Pedro 472, 15-VI-1941 (LISI); Santa Mata de Penaguião, Casal da Gaivosa, A. Rozeira, A. Serra, Bernardino \& Carlos, 2-III-1974 (PO 27046); Tabuaço, Quinta do Seixo, A. Rozeira, A. Serra, Bernardino \& Carlos, 3-III-1974 (PO 27074); entre Almendra e Castelo Melhor, Serro de S. Gabriel, G. Pedro 2037, 2-VI-1941 (LISI); Figueira de Castelo Rodrigo, Barca d'Alva, Quinta da Pedrica, A. Rozeira, D. Barreto, G. Costa \& A. Serra, 9-I-1971 (PO 27041); Freixo de Espada-á-Cinta, Poiares, Quinta do Saltinho, G. Pedro 758, 27-IV-1941 (LISI).

BEIRA TRANSMONTANA: Almeida, Vale de Marcos, A.R. da Cunha, VII-1884 (LISU, P-2623).

DOURO LITORAL: Vendas Novas, E. Johnston, 23-II-1890 (PO 2794); Alfena, Ponte Ferreira (arred. do Porto), E. Johnston, VII-1894 (COI); Marco de Canavezes, Vila Boa de Quires, Remungoso, no caminho para a Torre, G. Costa, 7-II-1971 (PO 27042); Porto, Ameeira, pr. Monte dos Burgos, J. Castro, 17-VII-1941 (PO 022 \& 6456); Fanzeres, Aguiar do Sousa, Rio Tinto e Porto, A. Lerso, s/data ( COI ).

BEIRA ALTA: Vil de Moinhos, Viseu, M. Ferreira, VII-1886 (COI); Viseu, margens do Dão, M. Ferreira, VII-1886 (COI), Mangualde, A. Moller, VII-1884 (COI); prox. Ponte da Atalhada, A. Moller, VII1886 (COI); arred. Oliveira do Conde, Petrofeira, A. Moller, VII1886 (COI).

BEIRA LITORAL: Cabrizes, Zorro, marg. do Mondego, J. Henriques, IV1880 (COI); arred. Coimbra, Brasfemes, M. Ferreira, VI-1889 (COI); Ponte da Murcela, Moura Morta, M. Ferreira, V-1882 \& V1895 (COI); Lousã, A. Moller, VI-1879 (COI); Gois, Ponte do Sotão, J. Henriques, VI-1883 (COI); Serra da Lousã, A. Moller, V1883 (COI).

BEIRA BAIXA: Covilha pr. Refúgio, A. Rozeira, K. Koepp \& G. Costa, 25-IV-1962 (PO 7108); Alpedrinha, Pucarinha, A.R. da Cunha, VII1883 (LISU, P-2625).

RIBATEJO: De Constância a Abrantes, J. Daveau, VI-1884 (COI).

Alto Alentejo: Castelo de Vide, Senhora de Penha, A.R. da Cunha, VI-1882 (LISU, P-2627); Marvão, Covões, A.R. da Cunha, VI-1882 (LISU, P-2626); Portalegre, Tapada dos Carteiros A.R. da Cunha, VI-1882 (LISU, P-2628); Vila Viçosa, Tapada Real, Monte das Pedras Escorregadias, A. Fernandes \& Sousa 603, V-1947 (COI); Tapada Real, Canto da Asseca, A. Fernandes \& Sousa 917, V-1947 (COI). 
Esta espécie parece ser frequente em solos graníticos ou xistosos, sobretudo nas bacias dos rios Douro, Vouga, Mondego, Tejo e alto Guadiana, em sítos frescos e um tanto sombrios.

Nos espécimes com folhas já adiantadas, os pelos glandulosos, que são sempre curtos e pouco densos, por vezes caducam em grande parte, pelo que não é de estranhar a sua inclusão em outras espécies do género, como, por exemplo, Ch. pteridioides, por botânicos menos experientes neste grupo de fetos. A existência deses pelos deu origem a que Mendonça e Vasconcellos supusessem tratar-se dum híbrido entre Ch. pteridioides e Ch. hispanica.

5. Ch. hispanica Mett. Abh. Senckenb. Naturf. Ges., 3: 74 (1859).

TRAS-OS-MONTES: Mirandela, Torre de D. Chama, a caminho de Valpaços, à Ponte da Pedra, P. Lopes \& G. Pedro 2762, 14-X-1941 (LISI); Mogadouro, S. Pedro J.R. dos Santos Junior, I-1925 (PO 2796 p.p.).

ALTO DOURO: Mirandela, Serra de Passos, crista do fragão do Soalheiro, G. Pedro 2797, 15-X-1941 (LISI); arred. de Mirandela, exp. S.W., F. Mendonça \& J. de Vasconcellos 370, 8-IV-1941 (LISI); Mirandela, Rozeira \& J. Castro, 29-III-1942 (PO 023); Vila Real, margem do rio Corgo, Rozeira \& J. Castro, 1-XII-1943 (PO 5203); Tua, a caminho de fiolhal, G. Barbosa \& M. Myre 3919, 10-V-1942 (LISI); Tralhariz, G. Barbosa \& F. Garcia 7413, 18-X-1944 (LISI); Ribalonga, G. Pedro 3270, 20-IV-1942 (LISI); Moncorvo, Cabeço do Baldoeiro, marg. direita do rio Sabor, J.R. Santos Junior, 25-III-1967 (PO 26969); Estremadouro, F. Mendonça \& J. de Vasconcellos 5303, 27-IV-1943 (LISI); Peso da Régua, Fonte de Ciderma, F. Mendonça \& J. de Vasconcellios 432-A, 11-IV-1941 (LISI); pr. Cachão da Valeira, G. Pedro 3419, 20-IV-1942 (LISI); Vila Nova de Foscoa, entre Vesúvio e Vargelas, ribeira da Teja, G. Barbosa \& M. Myre 4884, 13-X-1942 (LISI); Castelo Melhor, S. Gabriel, exp. S., G. Pedro 2040, 27-VI-1941 (LISI); Seixo do Vieiro, Urros, P. Lopes \& G. Pedro 630, 27-IV-1941 (LISI); Ligares, Fragão do Candedo, G. Barbosa \& M. Myre \& G. Pedro 3573, 30-IV-1942 (LISI); Freixo de Espada-à-Cinta, a $5 \mathrm{~km}$. de Barca d'Alva, A. Rozeira, K. Koepp \& G. Costa, 14-XII-1961 (PO 7107); Pencedo Durão, A. Taborda de Morais 3898, 29-IX-1938 (COI) .

DOURO LITORAL: Santo Tirso, Caldas da Saude, Barros Carneiro, 16XII-1944 (PO 5012); pr. a Valongo, naserra, J. Castro, 10-VI-1936 (PO 024); Queira, G. Costa \& M. Araujo, 21-III-1963 (PO 26968); Valongo, a sul, marg. rio Ferreira, E. Johnston, 30-VIII-1891 (PO 3562) \& 5-V-1901 (PO 2795), e A. Rozeira, 11-IV-1936 (PO 7110); entre a estação e a ponte, A. Rozeira, Martins d'Alte \& J. Castro, 9-VIII-1949 (PO 3783), Campo, G. Costa \& J. Araujo, 16-V-1965 (PO 7109); Paredes, Aguiar do Sousa, Fuandalva, J. Castro \& G. Costa, 6-VII-1955 (PO 5991); Castelo de Aguiar do Sousa, J. Castro \& G. Costa, 12-IV-1956 (PO 6216); Vila Nova de Gaia, Crestuma, E. Johnston, 2-X-1888 (PO 2800); Crestuma, Fontinha, J. Castro \& G. Costa, 17-III-1958 (PO 7011); Gondomar, foz do Sousa, Esposade, G. Costa \& J. Araujo, 28-V-1966 (PO 7110); Penafiel, ribeira de Couce, pr. a Sebolinho, D. Barreto, G. Costa \& A. Serra, 3-IV-1971 (PO 27043); arred. do Porto, A. Luso, s/data (COI).

BEIRA TRASMontanA: Mata de Lobos, Navarra, F. Garcia \& J. Pedrogão 6322, 13-IV-1944 (LISI); estrada de Figueira de Castelo Rodrigo à Guada, ao km. 145, próx. ao rio, A. Rozeira, K. Koepp \& G. Cos- 
ta, 16-XII-1960 (PO 7106); Almeida, rio Coa, M. Ferreira, s/data ( $\mathrm{COI}$ ).

BEIRA ALTA: Alvarenga, a $2 \mathrm{~km}$. para Arouca, J. Matos \& A. Diniz 9727, 9-VII-1966 (COI); Viseu, Serra de Santa Luzia, M. Ferreira, VII-1886 (COI); Serra do Caramulo, Cabeça do Cão, M. Ferreira,VI1884 (COI).

BEIRA LITORAL: Oliveira do Conde, A. Moller, VII-1886 (COI); pr. Conimbricam, Welwitsch, VIII-1848 (LISU, P-2635 \& P-2636); Serra do Dianteiro, M. Ferreira, IX-1877 (COI); Dianteiro, M. Ferreira, VII-1883 (LISI: LISU, P- 2630 \& P-2634); Valbom, A. Moller, VII1883 (COI); Penacova, Penedo do Castro, A. Reis Moura, 29-XI-1965 (COI); Lousã, G. Franqueira, VII-1894 (COI); Serra da Lousã, pr. da vila A. Moller, VI-1879 (COI); Lousã, Na Senhora da Piedade, U. Beua 2050, 5-VI-1963 (COI); Serra da Lousã, A. Moller, V-1883 (COI) e pr. Pontão da Cerdeira, C.N. Tavare, 13-XII-1953 (LISU, $\mathrm{P}-55572)$.

BEIRA BAIXA: Castelo Branco, Feiteira, A.R. da Cunha, VI-1881 (LISU, P-2637); Zibreira, R. Palhinha, IV-1930 (LISU, P-2631); Ferreira do Zêzere, Machial, M. Ferreira, VI-1914 (COI); Lagar do Gato, I. Nogueira \& al. 10963, 27-II-1970 (COI); Vila Velha de Rodão, a caminho de Fratel, A. Fernandes \& al. 7699, 26-III-1961 (COI).

ALto ALENTEJO: Beirã, arredores, R. Palhinha \& F. Mendes, V-1913 (LISU, P-2632); Portalegre, A. Moller, VI-1891 (COI); Alegrete, Sesmarias (Touril), A.R.F. Raimundo, 12-VI-1952 (LISI); Azinhalinho, pr. estrada de Mourão, R. Palhinha \& F. Mendes, V-1909 (LISU, P-2633); Belver, Panha dos Abotoreiros, J. Simões, VIII-1886 (COI).

Espécie ibero-norteafricana de sítios secos, de preferência insolados, frequentemente de exposição a Sul, com solos de características xistosas ou cristalofílicas, delgados, em fendas ou intersticios de rochas.

6. Ch. marantae (L.) Domin, Biblioth. Bot. (Stuttgart), 20: 133, adnot. 1 (1915).

TRAS-OS-MONTES: Vinhais, Ousilhão, entre Lombeira de Fontrelas e Tula, P. Silva, B. Rainha \& J. Martins 7833 (LISE); Bragança, Donai, Serro 3ํ, Sardoal de Donai, P. Silva, B. Rainha \& J. Martins 7852 (LISE); Donai, Cabeço do Joguinho, P. Silva \& B. Rainha 7186, 1963 (LISE) e P. Silva, B. Rainha \& J. Martins 7414 (LISE); Macedo de Cavaleiros, pr. Lagoa, abaixo do Lombo dos Pojos, P. Silva, B. Rainha \& J. Martins 7685 \& 7739 (COI \& LISE); Mogadouro, Soutelo, pr. Piçarrão, P. Silva, B. Rainha \& J. Martins 7808 (LISE).

Esta espécie, primeiramente colhida em Portugal, próximo de Bragança, em 1963 por P. Silva e colaboradores, encontra-se, segundo este autor, confinada às fendas das rochas serpentínicas do Nordeste transmontano português.

7. Ch vellea (Aiton) F. Muell., Fragm. Phytogr. Austral., 5: $123(1866)$.

ALTO DOURO: Vale do Tedo, P. Lopes \& G. Pedro 2499, 3-X-1941 (LISI); Freixo de Espada-à-Cinta, Poiares, Quinta da Foz do Ribeiro do Mosteiro, G. Pedro 1799, 22-VI-1941 (LISI); entre a Quinta da 
Malhadinha e a ribeira do Mosteiro, G. Barbosa \& F. Garcia 8536, 2-V-1946 (LISI); Poiares, margens do Douro, P. Lopes \& G. Pedro 714, 26-IV-1941 (LISI) e G. Barbosa, M. Myre \& G. Pedro 3691 , 1-V-1942 (LISI); Barca d'Alva, margem esquerda do rio Agueda, F. Mondonça \& J. de Vasconcellos 6187, 6-IV-1944 (LISI).

ESTREMADURA: In rupestribus excelsis de Serra da Arrabida, 1848-1849 (LISU, P-2606); in rupestribus editioribus de Serra da Arrabida, Welwitsch, IV-1847 (LISU, P-2607).

BAIXO ALENTJO: Mertola, A. Moller, IV-1888 (COI \& LISU, P- 1688); margem da ribeira de Oeiras, próx. da ponte, M. Bravo Lima 283, 5-IV-1958 (LISI).

ALGARVE: Silves, S. Bartolomeu de Messines, Barrocal da Gralheira, L. Sobrinho \& F. Augusto, 23-IV-1968 (LISU, P-66722); Albufeira, Serra da Gralheira, C. Romariz \& E.J. Mendes, 13-III-1953 (LISU, P-55235); Barrocal da Gralheira, R. Fernandes \& al. 10486, 23IV-1968 (COI); Tavira, Almargem,E. da Veiga, s/data (COI).

Espécie acentuadamente xerofílica, preferindo, por isso, locais bastante secos e insolados, em fendas de rochas xistosas ou calcárias.

Comunicación presentada a la Reunión

Internacional de Pteridología (Algeciras, 1980) 
M. L. ROCHA

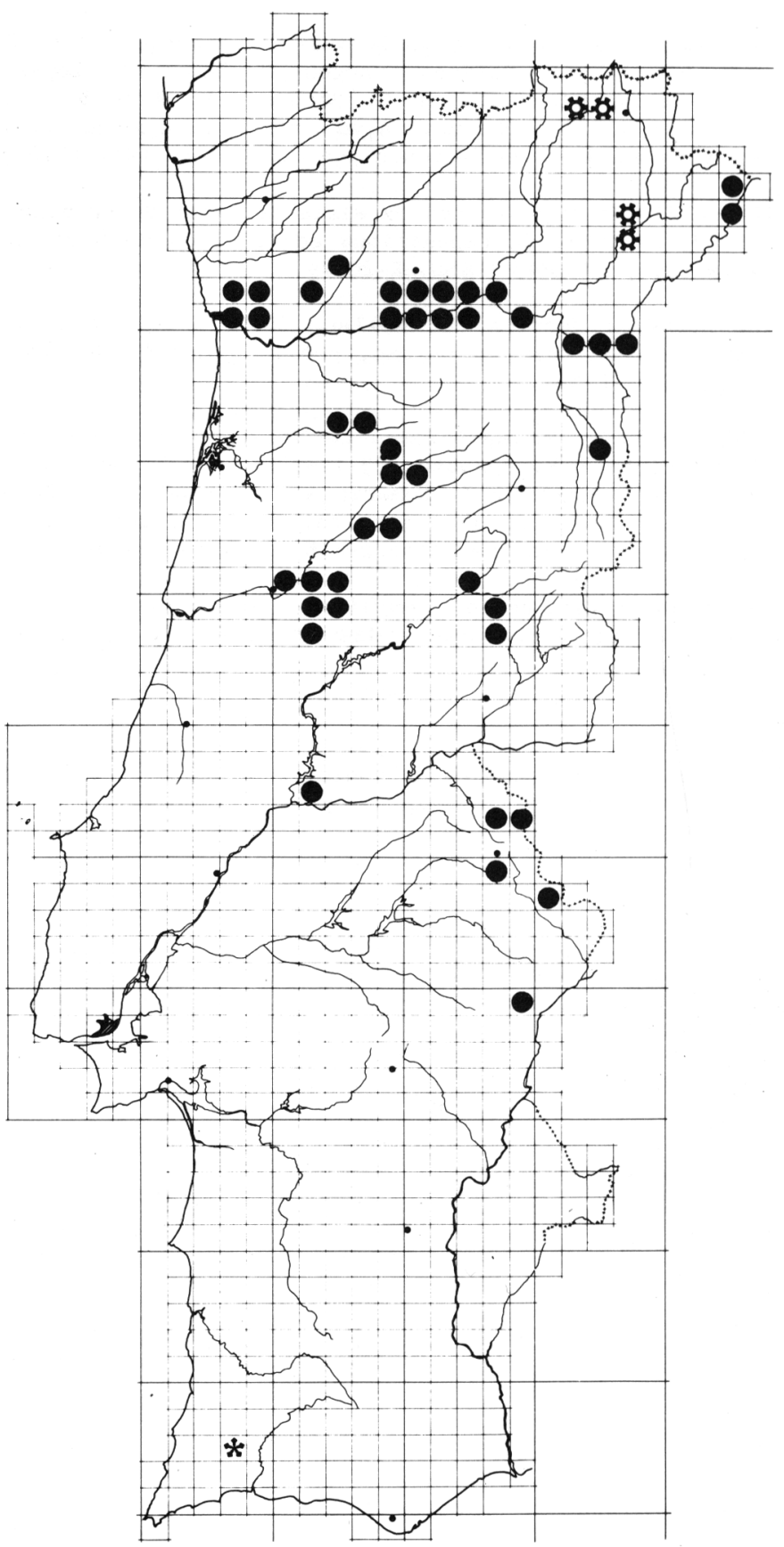

- Cheilanthes tinaei, $*$ Cheilanthes guanchica, marantae em Portugal. 


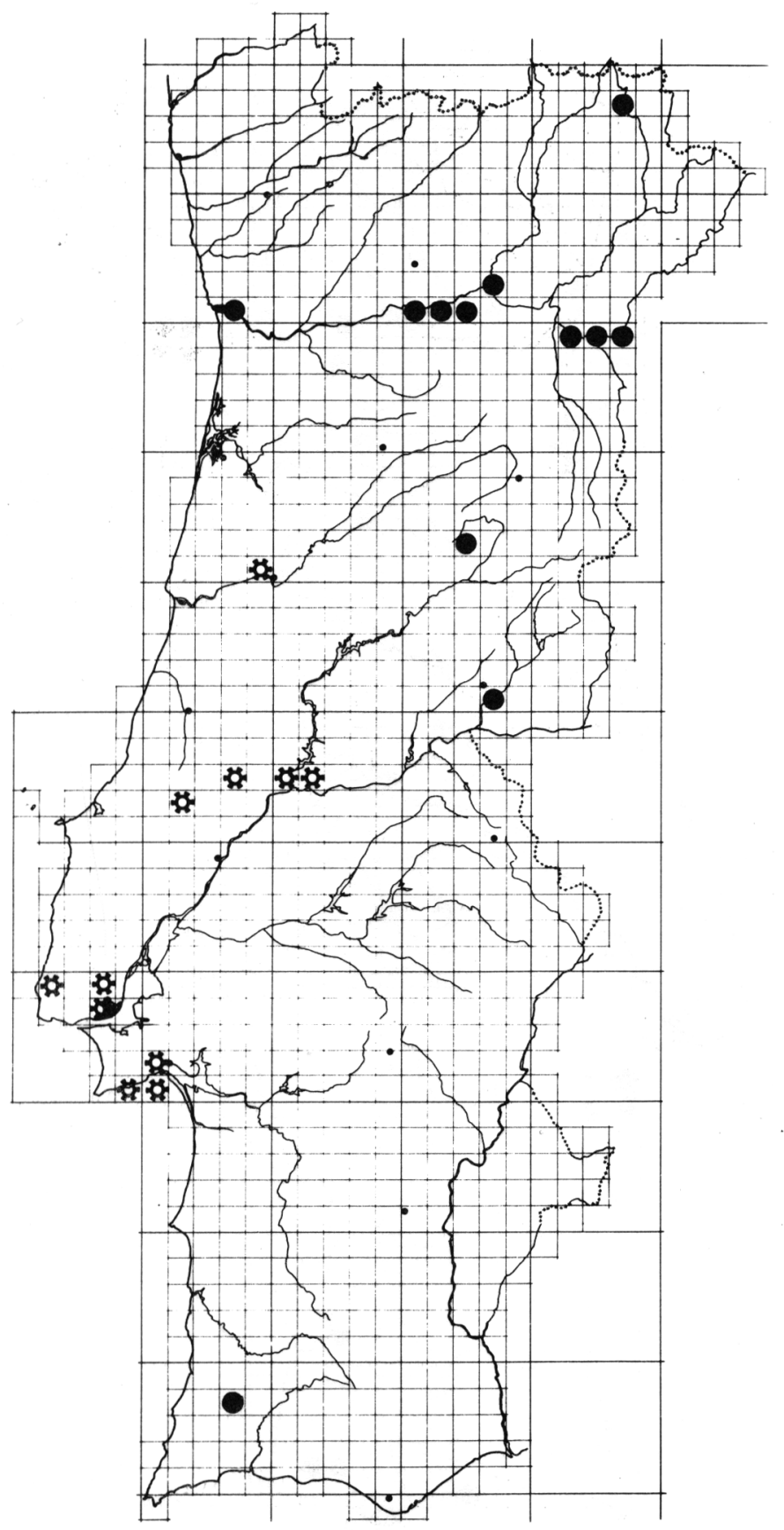

- Cheilanthes maderensis, Cheilanthes pteridioides em Portugal 


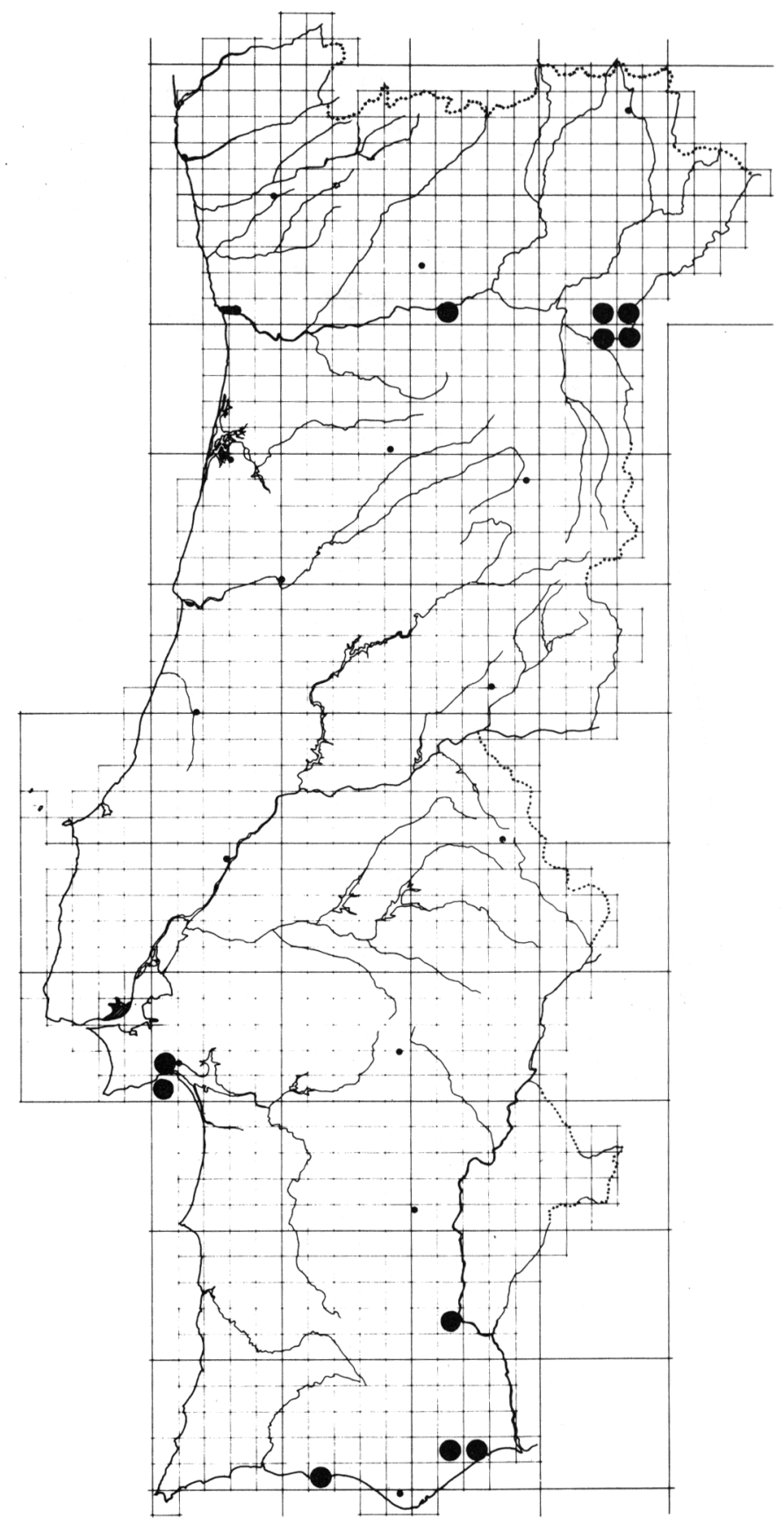

- Cheilanthes vellea em Portugal. 


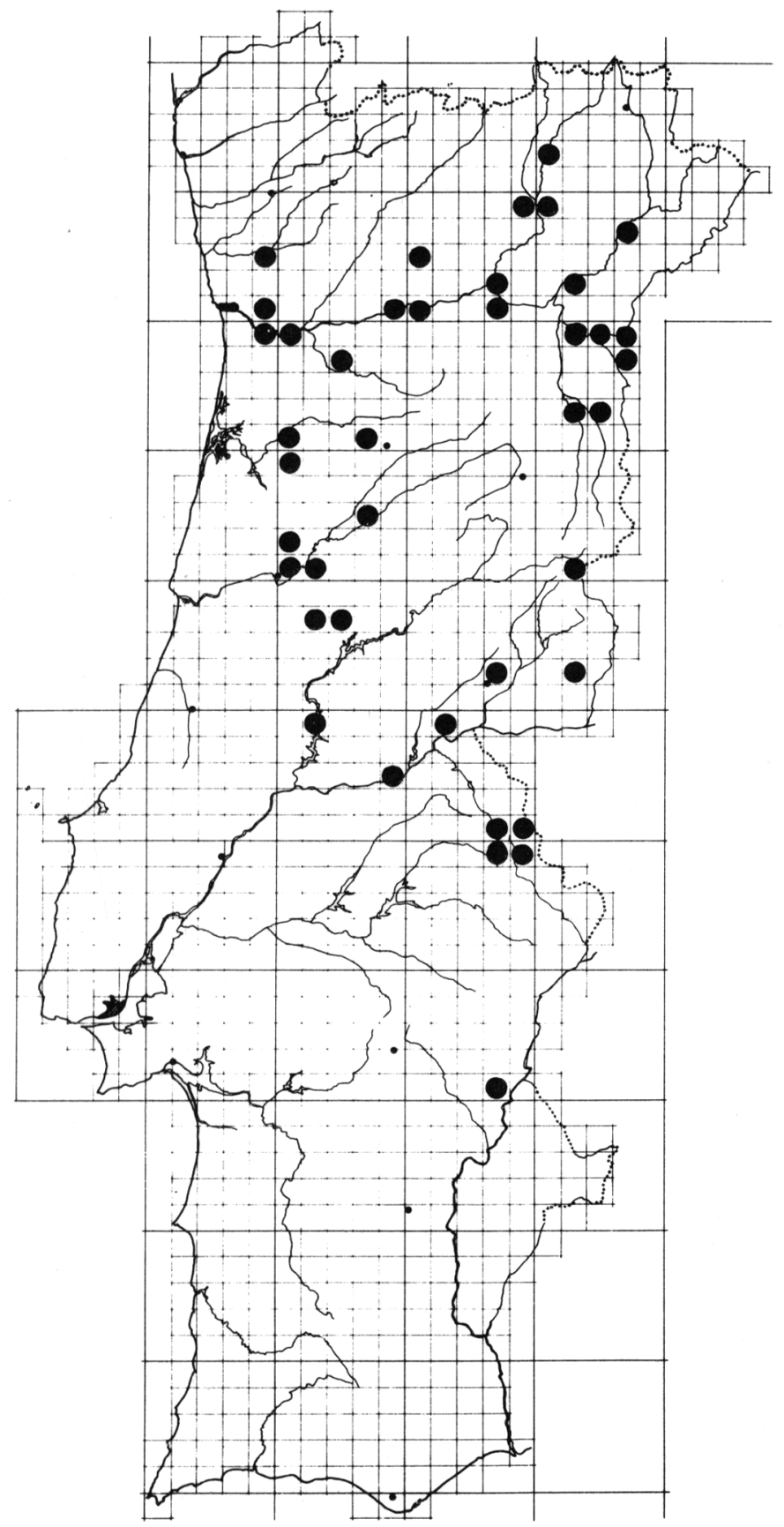

Cheilanthes hispanica em Portugal. 